\title{
A Comparative Study Between Laplace Transform and Two New Integrals "ELzaki" Transform and "Aboodh" Transform
}

\author{
Abdelbagy A. Alshikh ${ }^{1,2}$, Mohand M. Abdelrahim Mahgob ${ }^{1,3}$ \\ ${ }^{1}$ Mathematics Department Faculty of Sciences and Arts-Almikwah, Albaha University, Albaha, Saudi Arabia \\ ${ }^{2}$ Mathematics Department Faculty of Education, Alzaeim Alazhari University, Khartoum, Sudan \\ ${ }^{3}$ Mathematics Department Faculty of Sciences, Omderman Islamic University, Khartoum, Sudan \\ Email address: \\ abd.elbagy@hotmail.com (A. A. Alshikh),mahgob10@hotmail.com (M. M. A. Mahgob)
}

To cite this article:

Abdelbagy A. Alshikh, Mohand M. Abdelrahim Mahgob. A Comparative Study Between Laplace Transform and Two New Integrals "ELzaki” Transform and “Aboodh” Transform. Pure and Applied Mathematics Journal. Vol. 5, No. 5, 2016, pp. 145-150. doi: 10.11648/j.pamj.20160505.11

Received: August 7, 2016; Accepted: August 20, 2016; Published: September 7, 2016

\begin{abstract}
In this paper we discuss some relationship between Laplace transform and the new two transform called ELzaki transform and Aboodh transform. We solve first and second order ordinary differential equations using both transforms, and show that ELzaki transform and Aboodh transform are closely connected with the Laplace transform.
\end{abstract}

Keywords: Laplace Transform, Elzaki Transform, Aboodh Transform, Differential Equation

\section{Introduction}

Integral transforms play an important role in many fields of science. In literature, integral transforms are widely used in mathematical physics, optics, engineering mathematics and, few others. Among these transforms which were extensively used and applied on theory and applications are: the variational iteration method, the homotopy perturbation method [5], the differential transform method [(2008)] and adomian decomposition method.

The Laplace transform has been effectively used to solve linear and non-linear ordinary and partial differential equations and is used extensively in electrical engineering. The Laplace transform reduces a linear differential equation to an algebraic equation, which can be solved by rules of algebra. The original differential equation can then be solved by applying the inverse Laplace transform

Tarig M. Elzaki and Sailh M. Elzaki in [1-4], showed the modified of Sumudu transform [6-10] or Elzaki transform was applied to partial differential equations, ordinary differential equations, system of ordinary and partial differential equations and integral equations. Elzaki transform is a powerful tool for solving some differential equations which cannot solve by Sumudu transform.

Aboodh Transform [11, 12] was introduced by Khalid Aboodh in 2013, to facilitate the process of solving ordinary and partial differential equations in the time domain. This transformation has deeper connection with the Laplace and Elzaki Transform. [8, 9].

The main objective is to introduce a comparative study to solve differential equations by using Laplace transform and Elzaki transform and a boodh transform. The plane of the paper is as follows: In section 2, we introduce the basic idea of Laplace transform, then, Elzaki Transform in 3, then, Aboodh Transform in 4, Application in 5 and conclusion in 6, respectively.

\section{Definitions and Standard Results}

\subsection{The Laplace Transform}

Definition: If $f(t)$ is a function defined for all positive values of $t$, then the Laplace Transform of is defined as

$$
L[f(t)]=F(s)=\int_{0}^{\infty} e^{-s t} f(t) d t
$$

provided that the integral exists. Here the parameter sis a real or complex number. The corresponding inverse Laplace 
transform is $L^{-1}[F(s)]=f(t)$. Here $f(t)$ and $F(s)$ are called as pair of Laplace transforms.

Theorem 1: Laplace transform is a linear transform. That is, if then

$$
L\left[c_{1} f_{1}(t)+c_{2} f_{1}(t)\right]=c_{1} L\left[f_{1}(t)\right]+c_{2} L\left[f_{1}(t)\right]
$$

where $c_{1}$ and $c_{2}$ are constants.

LAPLACE transform of some functions:

$$
\begin{gathered}
L(1)=\frac{1}{s}, L\left(t^{n}\right)=\frac{n !}{s^{n+1}}, L\left(e^{a t}\right)=\frac{1}{s-a} \\
L(\sin (a t))=\frac{a}{s^{2}+a^{2}}, L(\cos (a t))=\frac{s}{s^{2}+a^{2}}
\end{gathered}
$$

LAPLACE transform of derivatives:

(i) $L\left[f^{\prime}(t)\right]=s F(s)-f(0)$

(ii) $E\left[f^{\prime \prime}(t)\right]=s^{2} F(s)-s f(0)-f^{\prime}(0)$

\subsection{Elzaki Transform}

Definition.

Elzaki Transform [2]. Given a function $f(t)$ defined for all $t \geq 0$, as follow:

$$
E[f(t), v]=T(v)=v \int_{0}^{t} f(t) e^{-\frac{t}{v}} d t, v \in\left(k_{1}, k_{2}\right)
$$

for all values of $s$, for which the improper integral converges Elzaki transform of some functions:

$$
\begin{aligned}
& E(1)=v^{2}, E\left(t^{n}\right)=n ! v^{n+2}, E\left(e^{a t}\right)=\frac{v^{2}}{1-a v} \\
& E(\sin (a t))=\frac{a v^{3}}{1+a^{2} v^{2}}, E(\cos (a t))=\frac{v^{2}}{1+a^{2} v^{2}} .
\end{aligned}
$$

Elzaki transform of derivatives:

(i) $E\left[f^{\prime}(t)\right]=\frac{T(u)}{u}-u f(0)$

(ii) $E\left[f^{\prime \prime}(t)\right]=\frac{T(u)}{u^{2}}-f(0)-u f^{\prime}(0)$

\subsection{Aboodh Transform}

Definition:

A new transform called the Aboodh transform defined for function of exponential order we consider functions in the set A, defined by:

$$
A=\left\{f(t): \exists M, k_{1}, k_{2}>0,|f(t)|<M e^{-v t}\right.
$$

For a given function in the set $\mathrm{M}$ must be finite number, $k_{1}, k_{2}$ may be finite or infinite. Aboodh transform which is defined by the integral equation

$$
A[f(t)]=K(v)=\frac{1}{v} \int_{0}^{\infty} f(t) e^{-v t} d t t \geq 0, \mathrm{k}_{1} \leq v \leq \mathrm{k}_{2}(3)
$$

Aboodh transform of some functions:

$$
\begin{gathered}
A(1)=\frac{1}{v^{2}}, A\left(t^{n}\right)=\frac{n !}{v^{n+2}}, A\left(e^{a t}\right)=\frac{1}{v^{2}-a v} \\
A(\sin (a t))=\frac{a}{v\left(v^{2}+a^{2}\right)}, A(\cos (a t))=\frac{1}{\left(v^{2}+a^{2}\right)}
\end{gathered}
$$

Aboodh transform of derivatives:

$$
\begin{gathered}
A\left[f^{\prime}(t)\right]= \\
v K(v)-\frac{f(0)}{v}, A\left[f^{\prime \prime}(t)\right]=v^{2} K(v)-\frac{f^{\prime}(0)}{v}-f(0) \\
A\left[f^{(n)}(t)\right]=v^{n} K(v)-\sum_{k=0}^{n-1} \frac{f^{(k)}(0)}{v^{2-n+k}}
\end{gathered}
$$

\section{Application}

Example 5.1 consider the first order differential equation

$$
\frac{d y}{d x}+y=0
$$

With the initial condition;

$$
y(0)=1
$$

Solution:

Applying the Laplace transform of both sides of Eq. (4),

$$
L\left\{\frac{d y}{d x}\right\}+L\{y\}=L\{0\}
$$

Using the differential property of Laplace transform Eq. (12) can be written as:

$$
s y(s)-y(0)+y(s)=0
$$

Using initial condition (5), Eq. (7) can be written as:

$$
y(s)=\frac{1}{s+1}
$$

The inverse Laplace transform of this equation is simply obtained as

$$
y(x)=e^{-x},
$$

Where $y(s)$ is the Laplace transform of the function $y(x)$ 2: Applying the Elzaki transform of both sides of Eq. (4),

$$
E\left\{\frac{d y}{d x}\right\}+E\{y\}=E\{0\}
$$

Using the differential property of Elzaki transform Eq.(10) can be written as:

$$
\frac{1}{v} E(y)-v y(0)+E(y)=0
$$

Using initial condition (5), Eq. (11) can be written as:

$$
E(y)=\frac{v^{2}}{1+v}
$$

The inverse Elzaki transform of this equation is simply obtained as

$$
y(x)=e^{-x}
$$

Where $E(y)$ Is the Elzaki transform of the function $y(x)$ 3: Applying the Aboodh transform of both sides of Eq. (4),

$$
\mathrm{A}\left\{\frac{d y}{d x}\right\}+\mathrm{A}\{y\}=A\{0\}
$$

Using the differential property of Aboodh transform 
Eq.(14) can be written as

$$
v A(y)-\frac{y(0)}{v}+A(y)=0
$$

Using initial condition (5), Eq. (15) can be written as:

$$
A(y)[v+1]=\frac{1}{v}, A(y)=\frac{1}{v[v+1]}
$$

The inverse Aboodh transform of this equation is simply obtained as

$$
y(x)=e^{-x}
$$

Where $A(y)$ is the Aboodh transform of the function $y(x)$

Example 5.2 solve the differential equation

$$
y^{\prime}+2 y=x
$$

With the initial condition;

$$
y(0)=1
$$

Solution: Applying the Laplace transform of both sides of Eq. (16),

$$
\mathrm{L}\left\{y^{\prime}\right\}+\mathrm{L}\{2 y\}=\mathrm{L}\{x\}
$$

Using the differential property of Laplace transform Eq.(16) can be written as

$$
s y(s)-y(0)+2 y(s)=\frac{1}{s^{2}}
$$

Using initial condition (17), Eq. (19) can be written as

$$
y(s)=\frac{s^{2}+1}{s^{2}(s+2)}
$$

The inverse Laplace transform of this equation is simply obtained as

$$
y(x)=-\frac{1}{4}+\frac{1}{2} x+\frac{5}{4} e^{-2 x}
$$

Where $y(s)$ is the Laplace transform of the function $y(x)$

2: Applying the Elzaki transform of both sides of Eq. (16),

$$
\mathrm{E}\left\{y^{\prime}\right\}+\mathrm{E}\{2 y\}=\mathrm{E}\{x\}
$$

Using the differential property of Elzaki transform Eq.(16) can be written as:

$$
\frac{1}{v} E(y)-v y(0)+2 E(y)=v^{3}
$$

Using initial condition (17), Eq. (23) can be written as

$$
\begin{gathered}
E(y)=\frac{\left(v^{3}+v\right) v}{1+2 v} \\
E(y)=\frac{1}{2} v^{3}+\frac{5}{4}\left(\frac{v^{2}}{1+2 v}\right)-\frac{1}{4} v^{2}
\end{gathered}
$$

The inverse transform of this equation is simply obtained as

$$
y(x)=\frac{1}{2} x+\frac{5}{4} e^{-2 x}-\frac{1}{4}
$$

3: Applying the Aboodh transform of both sides of Eq. (16),

$$
\mathrm{A}\left\{y^{\prime}\right\}+\mathrm{A}\{2 y\}=\mathrm{A}\{x\}
$$

Using the differential property of Aboodh transform Eq.(16) can be written as:

$$
v A(y)-\frac{y(0)}{v}+2 A(y)=A(x)
$$

Using initial condition (17), Eq. (27) can be written as

$$
\begin{gathered}
v A(y)-\frac{1}{v}+2 A(y)=\frac{1}{v^{3}}, A(y)[v+2]=\frac{1}{v^{3}}+\frac{1}{v} \\
A(y)=\frac{1+v^{2}}{v^{3}[v+2]}
\end{gathered}
$$

The inverse Aboodh transform of this equation is simply obtained as

$$
y(x)=\frac{1}{2} x+\frac{5}{4} e^{-2 x}-\frac{1}{4}
$$

Example 5.3 Let us consider the second-order differential equation

$$
y^{\prime \prime}+y=0
$$

With the initial condition;

$$
y(0)=y^{\prime}(0)=1
$$

Solution: Applying the Laplace transform of both sides of Eq. (30),

$$
\mathrm{L}\left\{y^{\prime \prime}\right\}+\mathrm{L}\{y\}=\mathrm{L}\{0\}
$$

Using the differential property of Laplace transform Eq.(32) can be written as

$$
s^{2} y(s)-s y(0)-y^{\prime}(0)+y(s)=0
$$

Using initial condition (31), Eq. (33) can be written as

$$
y(s)=\frac{s+1}{s^{2}+1}
$$

The inverse Laplace transform of this equation is simply obtained as

$$
y(x)=\cos x+\sin x
$$

Where $y(s)$ is the Laplace transform of the function $y(x)$ 2: Applying the Elzaki transform of both sides of Eq. (30),

$$
\mathrm{E}\left\{y^{\prime \prime}\right\}+\mathrm{E}\{y\}=\mathrm{E}\{0\}
$$

Using the differential property of Elzaki transform Eq.(36) can be written as

$$
\frac{1}{v^{2}} E(y)-1+E(y)-v=0
$$

Using initial condition (31), Eq. (37) can be written as

$$
E(y)=\frac{v^{2}}{v^{2}+1}+\frac{v^{3}}{v^{2}+1}
$$


The inverse Elzaki transform of this equation is simply obtained as

$$
y(x)=\cos x+\sin x
$$

3: Applying the Aboodh transform of both sides of Eq. (30),

$$
\mathrm{A}\left\{y^{\prime \prime}\right\}+\mathrm{A}\{y\}=\mathrm{A}\{0\}
$$

Using the differential property of Aboodh transform Eq.(40) can be written as:

$$
v^{2} A(y)-\frac{y^{\prime}(0)}{v}-y(0)+A(y)=0
$$

Using initial condition (31), Eq. (41) can be written as

$$
\begin{aligned}
v^{2} A(y)-\frac{1}{v}-1+A(y) & =0, A(y)\left[v^{2}+1\right]=1+\frac{1}{v} \\
A(y) & =\frac{v+1}{v\left[v^{2}+1\right]}
\end{aligned}
$$

The inverse Aboodh transform of this equation is simply obtained as

$$
y(x)=\cos x+\sin x
$$

Example 5.4 Consider the second-order differential equation

$$
y^{\prime \prime}-3 y^{\prime}+2 y=0
$$

With the initial condition;

$$
y(0)=1, y^{\prime}(0)=4
$$

Solution: Applying the Laplace transform of both sides of Eq. (44),

$$
\mathrm{L}\left\{y^{\prime \prime}\right\}-\mathrm{L}\left\{3 y^{\prime}\right\}+\mathrm{L}\{2 y\}=\mathrm{L}\{0\}
$$

Using the differential property of Laplace transform Eq.(46) can be written as

$$
s^{2} y(s)-s y(0)-y^{\prime}(0)-3(s y(s)-y(0))+2 y(s)=0(47)
$$

Using initial condition (45), Eq. (47) can be written as

$$
y(s)=\frac{s+1}{s^{2}-3 s+2}
$$

Then take the inverse of Laplace transform we get

$$
\mathrm{y}(\mathrm{x})=-2 e^{t}+3 e^{2 t}
$$

Where $y(s)$ is the Laplace transform of the function $y(x)$ 2: Applying the Elzaki transform of both sides of Eq. (44),

$$
\mathrm{E}\left\{y^{\prime \prime}\right\}-\mathrm{E}\left\{3 y^{\prime}\right\}+\mathrm{E}\{2 y\}=\mathrm{E}\{0\}
$$

Using the differential property of Elzaki transform Eq.(50) can be written as

$$
E(y)=\frac{v^{2}(v+1)}{(2 v-1)(v-1)}=v^{2}\left[\frac{2}{v-1}-\frac{3}{2 v-1}\right]
$$

$$
E(y)=\frac{-2 v^{2}}{1-v}+\frac{3 v^{2}}{1-2 v}
$$

Then take the inverse of ELzaki transform we get

$$
\mathrm{y}(\mathrm{x})=-2 e^{t}+3 e^{2 t}
$$

3: Applying the Aboodh transform of both sides of Eq. (44),

$$
\mathrm{A}\left\{y^{\prime \prime}\right\}-\mathrm{A}\left\{3 y^{\prime}\right\}+\mathrm{A}\{2 y\}=\mathrm{A}\{0\}
$$

Using the differential property of Aboodh transform Eq.(53) can be written as:

$$
v^{2} A(y)-\frac{y^{\prime}(0)}{v}-y(0)-3 v A(y)+\frac{y(0)}{v}+2 A(y)=0
$$

Using initial condition (45), Eq. (54) can be written as

$$
\left(v^{2}-3 v+2\right) A(y)=\frac{3}{v}+1, \text { then } \mathrm{A}(\mathrm{y})=\frac{v+1}{v(v-2)(v-1)}
$$

Then take the inverse of Aboodh transform we get

$$
\mathrm{A}(\mathrm{x})=-2 e^{t}+3 e^{2 t}
$$

Example 5.5

Solve the differential equation:

$$
y^{\prime \prime}-3 y^{\prime}+2 y=4 e^{3 t}
$$

With the initial condition;

$$
y(0)=-3, y^{\prime}(0)=5
$$

Solution:

Applying the Laplace transform of both sides of Eq. (57),

$$
\mathrm{L}\left\{y^{\prime \prime}\right\}-\mathrm{L}\left\{3 y^{\prime}\right\}+\mathrm{L}\{2 y\}=\mathrm{L}\left\{4 e^{3 t}\right\}
$$

Using the differential property of Laplace transform Eq.(59) can be written as

$$
s^{2} y(s)-s y(0)-y^{\prime}(0)-3(s y(s)-y(0))+2 y(s)=\frac{4}{s-3}(60)
$$

Using initial condition (58), Eq. (60) can be written as

$$
y(s)=\frac{4}{(s-1)(s-2)(s-3)}-\left[\frac{3 s-14}{(s-1)(s-2)}\right]
$$

Then take the inverse of Laplace transform we get

$$
y(t)=4 e^{2 t}-9 e^{t}+2 e^{3 t}
$$

Where $y(s)$ is the Laplace transform of the function $y(x)$ 2: Applying the Elzaki transform of both sides of Eq. (59),

$$
\mathrm{E}\left\{y^{\prime \prime}\right\}-\mathrm{E}\left\{3 y^{\prime}\right\}+\mathrm{E}\{2 y\}=\mathrm{E}\left\{4 e^{3 t}\right\}
$$

Using the differential property of Elzaki transform Eq.(63) can be written as

$$
\frac{1}{v^{2}} T(v)+3-5 v-3\left[\frac{T(v)}{v}+3 v\right]+2 T(v)=\frac{4 v^{2}}{1-3 v}
$$




$$
\left[\frac{1}{v^{2}}-\frac{3}{v}+2\right] T(v)=\frac{4 v^{2}}{1-3 v}+14 v-3
$$

Then,

$$
T(v)=v^{2}\left[\frac{4}{1-2 v}-\frac{2}{1-3 v}-\frac{9}{1-v}\right]
$$

Inverting to find solution in the form

$$
y(t)=4 e^{2 t}-9 e^{t}+2 e^{3 t}
$$

3: Applying the Aboodh transform of both sides of Eq. (57),

$$
\mathrm{A}\left\{y^{\prime \prime}\right\}-\mathrm{A}\left\{3 y^{\prime}\right\}+\mathrm{A}\{2 y\}=\mathrm{A}\left\{4 e^{3 t}\right\}
$$

Using the differential property of Aboodh transform Eq.(66) can be written as:

$$
v^{2} y(v)-\frac{y^{\prime}(0)}{v}-y(0)-3 v y(v)+\frac{y(0)}{v}+2 y(v)=\frac{4}{v^{2}-3 v}
$$

Using initial condition (58), Eq. (67) can be written as

$$
y(v)=\frac{4}{v(v-1)(v-2)(v-3)}-\frac{3}{(v-1)(v-2)}+\frac{14}{v(v-1)(v-2)}
$$

Inverting to find solution in the form

$$
y(t)=4 e^{2 t}-9 e^{t}+2 e^{3 t}
$$

Example 5.6:

Find the solution of the following initial value problem

$$
y^{\prime \prime}+4 y=9 x
$$

With the initial condition,

$$
y(0)=0, y^{\prime}(0)=7
$$

Solution: Applying the Laplace transform of both sides of Eq. (70),

$$
\mathrm{L}\left\{y^{\prime \prime}\right\}+\mathrm{L}\{4 y\}=\mathrm{L}\{9 x\}
$$

Using the differential property of Laplace transform Eq.(72) can be written as

$$
s^{2} y(s)-s y(0)-y^{\prime}(0)+4 y(s)=\frac{9}{s^{2}}
$$

Using initial condition (71), Eq. (73) can be written as

$$
\left(s^{2}+4\right) y(s)=\frac{9}{s^{2}}+7 \text { so, } y(s)=\frac{7}{s^{2}+4}+\frac{9}{s^{2}\left(s^{2}+4\right)} \text { so, }(74)
$$

Inverting to find the solution in the form:

$$
y(x)=\frac{9}{4} x+\frac{19}{8} \sin 2 x
$$

Where $y(s)$ is the Laplace transform of the function $y(x)$ 2: Applying the Elzaki transform of both sides of Eq. (70),

$$
\mathrm{E}\left\{y^{\prime \prime}\right\}+\mathrm{E}\{4 y\}=\mathrm{E}\{9 x\}
$$

Using the differential property of Elzaki transform Eq.(76) can be written as

$$
\begin{gathered}
\frac{1}{v^{2}} T(v)-7 v+4 T(v)=9 v^{3}, \text { or } T(v)=\frac{9 v^{5}}{1+4 v^{2}}-\frac{7 v^{3}}{1+4 v^{2}}= \\
3 v^{2}+\frac{2 v^{3}}{1+4 v^{2}}
\end{gathered}
$$

Inverting to find the solution in the form:

$$
y(x)=\frac{9}{4} x+\frac{19}{8} \sin 2 x .
$$

3: Applying the Aboodh transform of both sides of Eq. (70),

$$
\mathrm{A}\left\{y^{\prime \prime}\right\}+\mathrm{A}\{4 y\}=\mathrm{A}\{9 x\}
$$

Using the differential property of Aboodh transform Eq.(78) can be written as:

$$
v^{2} k(v)-\frac{y^{\prime}(0)}{v}-y(0)+4 k(v)=\frac{9}{v^{3}}
$$

Using initial condition (71), Eq. (79) can be written as

$$
k(v)\left[v^{2}+4\right]=\frac{9}{v^{3}}+\frac{7}{v}
$$

$$
\text { So, } k(v)=\frac{7}{v\left(v^{2}+4\right)}+\frac{9}{v^{3}\left(v^{2}+4\right)}=\frac{7}{v\left(v^{2}+4\right)}+\frac{9}{v}\left[\frac{\frac{1}{4}}{v}-\frac{\frac{1}{4} v}{v^{2}+4}\right]
$$

Inverting to find the solution in the form

$$
y(x)=\frac{9}{4} x+\frac{19}{8} \sin 2 x
$$

\section{Conclusion}

The main goal of this paper is to conduct a comparative study between Laplace transform and new integrals "Elzaki transform" and "a boodh transform". The three methods are powerful and efficient. Elzaki transform and a boodh transform is a convenient tool for solving differential equations in the time domain without the need for performing an inverse Elzaki transform and inverse a boodh transform and the connection of Elzaki transform and a boodh transform with Laplace transform goes much deeper.

\section{References}

[1] Tarig M. Elzaki, (2011), The New Integral Transform "Elzaki Transform" Global Journal of Pure and Applied Mathematics, ISSN 0973-1768, Number 1, pp. 57-64.

[2] Tarig M. Elzaki\&Salih M. Elzaki, (2011), Application of New Transform "Elzaki Transform" to Partial Differential Equations, Global Journal of Pure and Applied Mathematics, ISSN 0973-1768, Number 1, pp. 65-70.

[3] Tarig M. Elzaki\&Salih M. Elzaki, (2011), On the Connections between Laplace and Elzaki transforms, Advances in Theoretical and Applied Mathematics, ISSN 0973-4554 Volume 6, Number 1, pp. 1-11.

[4] Tarig M. Elzaki\&Salih M. Elzaki, (2011), On the Elzaki Transform and Ordinary Differential Equation With Variable Coefficients, Advances in Theoretical and Applied Mathematics. ISSN 0973-4554 Volume 6, Number 1, pp. 1318. 
[5] D. D. Ganji, The applications of He's homotopy perturbation method to nonlinear equation arising in heat transfer, Physics Letters A, 335 (2006): 337-341.

[6] A. Kilicman and H. E. Gadain. (2009), An application of double Laplace transform and Sumudu transform, Lobachevskii J. Math. 30 (3), pp. 214-223. http://dx.doi.org/10.1134/s1995080209030044

[7] J. Zhang, (2007), A Sumudu based algorithm $\mathrm{m}$ for solving differential equations, Comp. Sci. J. Moldova 15 (3), pp - 303313.

[8] Hassan Eltayeb and AdemKilicman, (2010), A Note on the Sumudu Transforms and differential Equations, Applied Mathematical Sciences, VOL, 4, no. 22, 1089-1098.
[9] Kilicman A. \& H. ELtayeb. (2010), A note on Integral transform and Partial Differential Equation, Applied Mathematical Sciences, 4 (3), PP. 109-118.

[10] Hassan ELtayeh and AdemKilicman, (2010), on Some Applications of a new Integral Transform, Int. Journal of Math. Analysis, Vol, 4, no. 3, 123-132.

[11] K. S. Aboodh, The New Integral Transform "Aboodh Transform" Global Journal of pure and Applied Mathematics, 9 (1), 35-43 (2013).

[12] K. S. Aboodh, Application of New Transform "Aboodh transform" to Partial Differential Equations, Global Journal of pure and Applied Math, 10 (2), 249-254 (2014). 